\title{
Real-Time Supernova Neutrino Burst Monitor at Super-Kamiokande
}

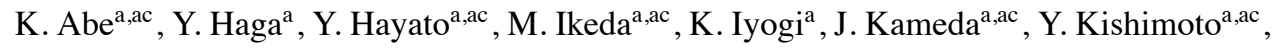
M. Miura ${ }^{\text {a,ac }}$, S. Moriyama ${ }^{\text {a,ac }}$, M. Nakahata ${ }^{\text {a,ac }}$, Y. Nakano $^{a}$, S. Nakayama ${ }^{\text {a,ac }}$, H. Sekiya a,ac, M. Shiozawa ${ }^{\mathrm{a}, \mathrm{ac}}$, Y. Suzuki ${ }^{\mathrm{a}, \mathrm{ac}}$, A. Takeda ${ }^{\mathrm{a}, \mathrm{ac}}, \mathrm{H}_{\text {. Tanaka }}{ }^{\mathrm{a}, \mathrm{ac}}$, T. Tomura $^{\mathrm{a}, \mathrm{ac}}$, K. Ueno $^{\mathrm{a}}$, R. A. Wendell ${ }^{\mathrm{a}, \mathrm{ac}}$, T. Yokozawa ${ }^{\mathrm{a}}$, T. Irvine ${ }^{\mathrm{b}}$, T. Kajita $^{\mathrm{b}, \mathrm{ac}}$, I. Kametani $^{\mathrm{b}}$, K. Kaneyuki $^{\mathrm{b}, \mathrm{ac}, 1}$, K. P. Lee ${ }^{b}$, T. McLachlan ${ }^{b}$, Y. Nishimura ${ }^{\text {b }}$, E. Richard ${ }^{b}$, K. Okumura ${ }^{\text {b,ac }}$, L. Labarga ${ }^{c}$,

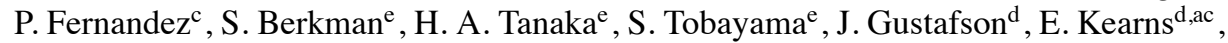
J. L. Raaf ${ }^{\mathrm{d}}$, J. L. Stone ${ }^{\mathrm{d}, \mathrm{ac}}$, L. R. Sulak ${ }^{\mathrm{d}}$, M. Goldhaber ${ }^{\mathrm{f}, 1}$, G. Carminati ${ }^{\mathrm{g}}$, W. R. Kropp ${ }^{\mathrm{g}}$, S. Mine ${ }^{\mathrm{g}}$, P. Weatherly ${ }^{\mathrm{g}}$, A. Renshaw ${ }^{\mathrm{g}}$, M. B. Smy ${ }^{\mathrm{g}, \mathrm{ac}}$, H. W. Sobel ${ }^{\mathrm{g}, \mathrm{ac}}$, V. Takhistov ${ }^{\mathrm{g}}$,

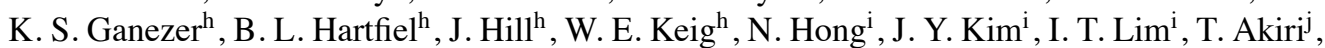

A. Himmel ${ }^{\mathrm{j}}$, K. Scholberg ${ }^{\mathrm{j}, \mathrm{ac}}$, C. W. Walter ${ }^{\mathrm{j}, \mathrm{ac}}$, T. Wongjirad $^{\mathrm{j}}$, T. Ishizuka $^{\mathrm{k}}$, S. Tasaka $^{\mathrm{l}}$, J. S. Jang ${ }^{\mathrm{m}}$, J. G. Learned ${ }^{\mathrm{n}}$, S. Matsuno ${ }^{\mathrm{n}}$, S. N. Smith ${ }^{\mathrm{n}}$, T. Hasegawa ${ }^{\mathrm{o}}$, T. Ishida ${ }^{\mathrm{o}}$, T. Ishii $^{\mathrm{o}}$,

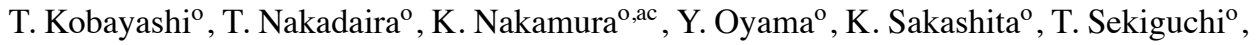

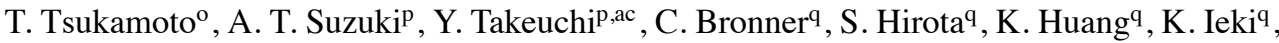

T. Kikawa ${ }^{q}$, A. Minamino ${ }^{q}$, A. Murakami ${ }^{q}$, T. Nakaya ${ }^{\text {q,ac }}$, K. Suzuki ${ }^{q}$, S. Takahashi ${ }^{q}$,

K. Tateishi ${ }^{\mathrm{q}}$, Y. Fukuda ${ }^{\mathrm{r}}$, K. Choi ${ }^{\mathrm{s}}$, Y. Itow ${ }^{\mathrm{s}}$, G. Mitsuka ${ }^{\mathrm{s}}$, P. Mijakowski ${ }^{\text {ah }}$, J. Hignight ${ }^{\mathrm{t}}$,

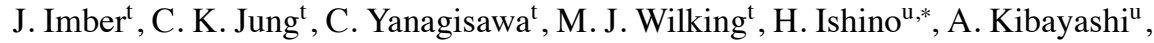

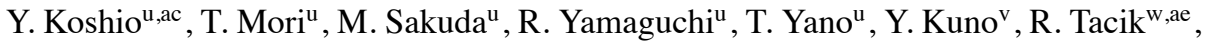
S. B. Kim ${ }^{\mathrm{x}}$, H. Okazawa ${ }^{\mathrm{y}}$, Y. Choi ${ }^{\mathrm{z}}$, K. Nishijima ${ }^{\mathrm{aa}}$, M. Koshiba ${ }^{\mathrm{ab}}$, Y. Suda ${ }^{\mathrm{ab}}$, Y. Totsuka ${ }^{\text {ab, },}$, M. Yokoyama ${ }^{\text {ab,ac }}$, K. Martens ${ }^{\text {ac }}$, Ll. Marti ${ }^{\text {ac }}$, M. R. Vagins ${ }^{\text {ac, g }, ~ J . ~ F . ~ M a r t i n ~}{ }^{\text {ad }}$, P. de Perio ${ }^{\text {ad }}$, A. Konaka ${ }^{\text {ae }}$, S. Chen ${ }^{\text {af }}$, Y. Zhang ${ }^{\text {af }}$, K. Connolly ${ }^{\text {ag }}$, R. J. Wilkes ${ }^{\text {ag }}$

(The Super-Kamiokande Collaboration)

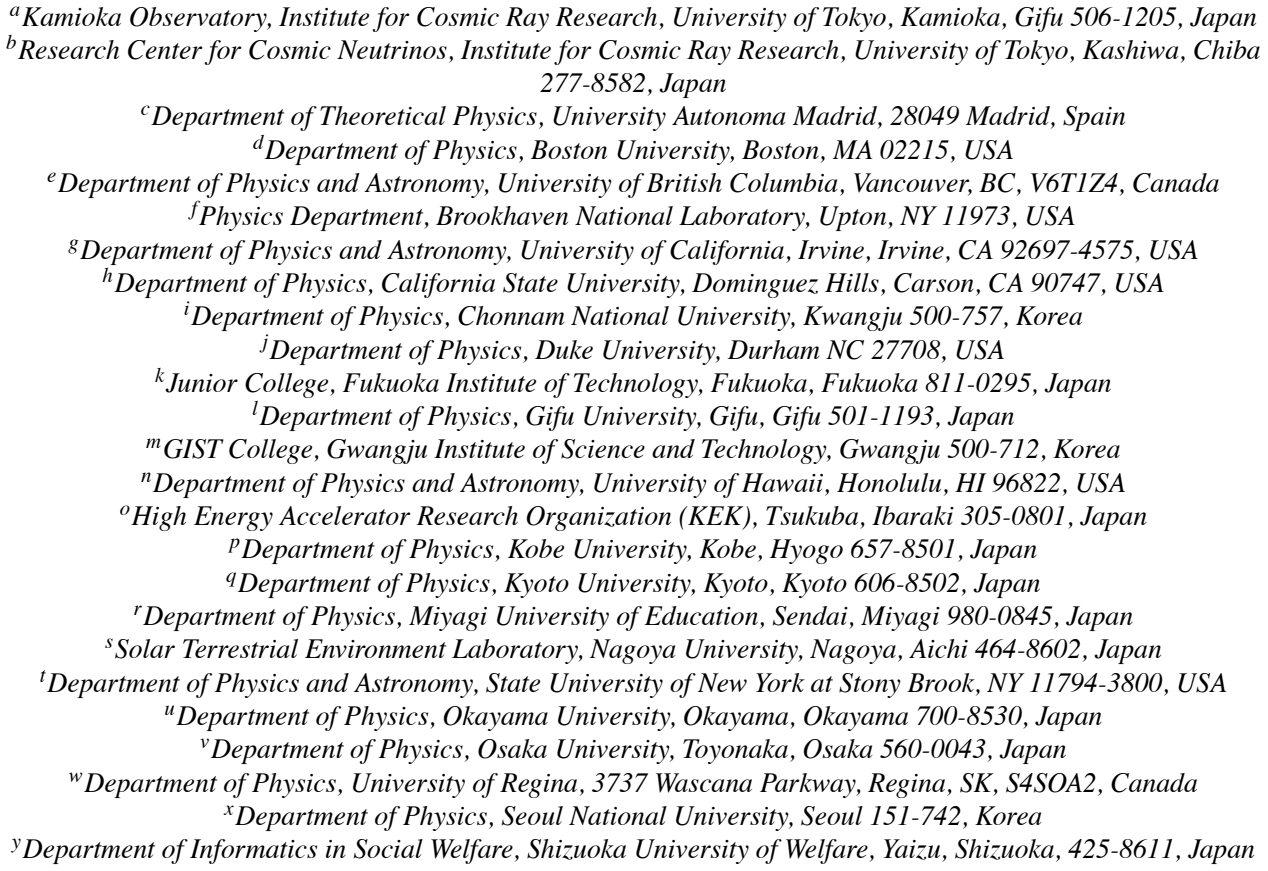




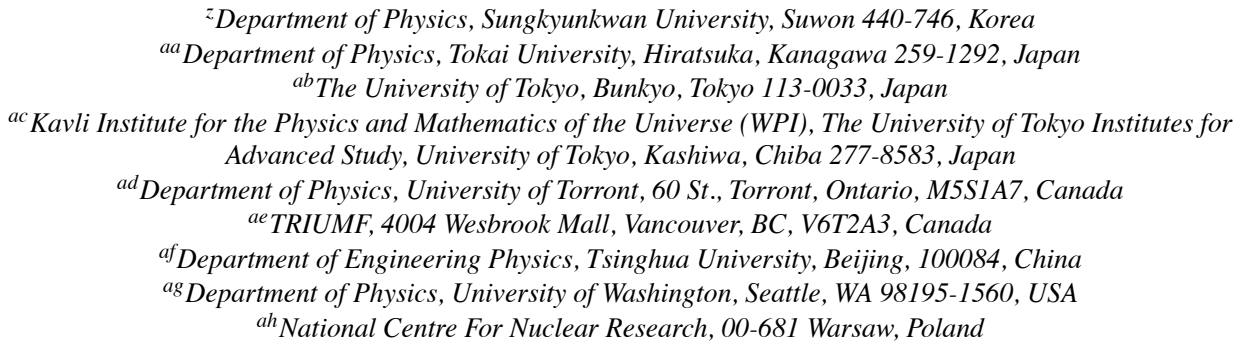

\begin{abstract}
We present a real-time supernova neutrino burst monitor at Super-Kamiokande (SK). Detecting supernova explosions by neutrinos in real time is crucial for giving a clear picture of the explosion mechanism. Since the neutrinos are expected to come earlier than light, a fast broadcasting of the detection may give astronomers a chance to make electromagnetic radiation observations of the explosions right at the onset. The role of the monitor includes a fast announcement of the neutrino burst detection to the world and a determination of the supernova direction. We present the online neutrino burst detection system and studies of the direction determination accuracy based on simulations at SK.
\end{abstract}

Keywords: Supernova, Neutrinos, Super-Kamiokande

\title{
1. Introduction
}

The detection of neutrinos from SN1987A opened a new era of neutrino astronomy [1]. Although the number of the detected neutrino events [2] was small, significant information about the supernova $(\mathrm{SN})$ explosion and neutrino properties was obtained [3]. The current generation of detectors are waiting for the next SN neutrino burst to accumulate event statistics much larger than those of SN1987A.

The SN burst neutrinos arrive at the Earth earlier than the electromagnetic radiation, since the neutrinos generated at the core of the explosion and emitted from the surface of the neutrinosphere travel at nearly the speed of light,while the shock waves propagating to the outside with velocity much slower than the neutrino velocity result in the emission of electromagnetic radiation [4]. The delay may depend on the structure of the envelope of the core as well as the surrounding stellar environment, and is expected to range between tens of minutes and tens of hours [5]. Therefore, the detection of the neutrino burst can generate a warning able to allow the astronomers to observe the radiation from the onset of the explosion. Such warning systems have been developed by several neutrino observatories [6] [7] as well as by the supernova early warning system (SNEWS) [8].

${ }^{*}$ Corresponding Author, Hirokazu Ishino, Department of Physics, Okayama University, scishino@s.okayama-u.ac.jp ${ }^{1}$ Deceased. 
It is also important to determine the SN direction using the neutrino signal: the direction information can guide optical instruments toward the SN explosion and enable observation of the onset of radiation. Among the neutrino detectors operating at present, Super-Kamiokande (SK) is the only detector able to determine the SN direction using neutrino events. We have developed a SN direction determination method by applying a maximum likelihood fit.

SK is the world's largest water Cherenkov detector located 1,000 m underground, inside a mountain in Kamioka, Gifu, Japan. The detector consists of 50,000 tons of ultra-pure water and about 13,000 photomultiplier tubes (PMTs). Based on the information of the yields and the arrival timing of Cherenkov photons for individual PMTs as well as the PMT locations, SK is able to measure the position, direction and energy of a neutrino event in real time. Details of the SK detector and its performance are described in [9]. In 2008, SK upgraded its readout system. The system has improved the data processing speed significantly, lowering the trigger energy threshold and dead time for the SN burst events [10].

We will describe the SN neutrino burst monitor at SK. In Section 2, we will describe details of the monitor system and its performance. We first describe SN models used in this report for performance evaluations. A Monte-Carlo (MC) simulation with the SN models is utilized for the estimation of the detection efficiency. We will explain the selection criteria to discriminate SN bursts and the main background in SK: radioactive decays caused by cosmic ray muon spallation [11]. In Section 3, we will describe a method to reconstruct the SN direction and studies of its performance.

\section{Real time supernova neutrino burst monitor}

\subsection{Supernova models}

We first describe the SN models we use in this report. We employ two models for the SN neutrino burst: the Wilson model [12] and the Nakazato model [13]. For the Nakazato model, we choose two parameter sets: $M=20, t_{\text {revive }}=200 \mathrm{msec}$ and $Z=0.02$ (NK1), and $M=13$, $t_{\text {revive }}=100 \mathrm{msec}$ and $Z=0.004$ (NK2), where $M$ is the progenitor mass in units of the solar mass, $t_{\text {revive }}$ is the shock revival time and $Z$ is the metallicity, respectively. We choose the first one for the SN1987A progenitor mass, which is about 20 times the solar mass. The model with the latter parameters gives the smallest neutrino fluxes in the Nakazato model. Both models provide time dependences of neutrino luminosities and energy spectra for $v_{e}, \bar{v}_{e}$ and $v_{x}$, for 18 seconds (20 seconds) for the Wilson (NK) model, where $v_{x}$ refers to the muon and tau types of neutrinos and anti-neutrinos. The anti-electron neutrino fluences of the models of Wilson, NK1 and NK2 are 16.0,9.8 and 9.4, respectively, in units of $10^{10} / \mathrm{cm}^{2}$ in the energy range of 7 to $50 \mathrm{MeV}$ at the distance of $10 \mathrm{kpc}$ without neutrino oscillation. We also take into account neutrino oscillations based on [14]. We assume $P_{H}=0$ in the parameterization of [14], which implies adiabatic transitions between electron and tau (anti-) neutrinos due to $\sin ^{2} 2 \theta_{13}=0.095 \pm 0.010$ [15]. In this report, we do not take into account collective effects. We use the cross sections [16] for the inverse beta decays, [17][18] for the charged current interactions to oxygen, and [19] for the electron elastic scatterings.

Monte-Carlo simulation samples are generated for the three SN models and three neutrino oscillation hypotheses by making use of the full SK detector MC simulator based on Geant3 in the SK inner detector volume (32.5-kton) in the energy range of 3 to $60 \mathrm{MeV}$ taking into account the trigger threshold curve. The calibration of the detector and its simulation are described in [20]. We apply the SK standard reconstruction program to the generated MC events to obtain the vertex 
Table 1: Numbers of expected events at SK in the 22.5-kton fiducial volume with the $7 \mathrm{MeV}$ total energy threshold for a SN burst with a distance of $10 \mathrm{kpc}$. We estimated these numbers using SK MC: we generate 3,000 ensembles of the MC samples, reconstructed the events with the SK standard reconstruction tool, applied the selection criteria, and then calculated the average numbers.

\begin{tabular}{cccccccccc}
\hline \hline & \multicolumn{3}{c}{ Wilson } & \multicolumn{3}{c}{ NK1 } & \multicolumn{3}{c}{ NK2 } \\
& no osc. & NH & IH & no osc. & NH & IH & no osc. & NH & IH \\
\hline $\bar{v}_{e}+p \rightarrow e^{+}+n$ & 4923 & 5667 & 7587 & 2076 & 2399 & 2745 & 1878 & 2252 & 2652 \\
$v_{e}+e^{-} \rightarrow v_{e}+e^{-}$ & 74 & 130 & 114 & 43 & 56 & 56 & 39 & 54 & 54 \\
$\bar{v}_{e}+e^{-} \rightarrow \bar{v}_{e}+e^{-}$ & 25 & 29 & 37 & 10 & 12 & 14 & 9 & 11 & 13 \\
$v_{x}+e^{-} \rightarrow v_{x}+e^{-}$ & 41 & 33 & 34 & 17 & 19 & 18 & 17 & 17 & 17 \\
$\bar{v}_{x}+e^{-} \rightarrow \bar{v}_{x}+e^{-}$ & 34 & 33 & 29 & 14 & 14 & 14 & 13 & 13 & 14 \\
$v_{e}+{ }^{16} \mathrm{O} \rightarrow e^{-}+X$ & 8 & 662 & 479 & 22 & 78 & 74 & 16 & 72 & 68 \\
$\bar{v}_{e}+{ }^{16} \mathrm{O} \rightarrow e^{+}+X$ & 64 & 196 & 531 & 27 & 48 & 70 & 20 & 41 & 64 \\
\hline total & 5169 & 6750 & 8811 & 2209 & 2626 & 2991 & 1992 & 2460 & 2882 \\
\hline \hline
\end{tabular}

position, the direction and the total energy of each event. In the $\mathrm{SN}$ monitor, we use events with total energy greater than $7 \mathrm{MeV}$ in the 22.5-kton fiducial volume, where the fiducial volume is defined as the volume whose surface is located $2 \mathrm{~m}$ inside from the surface of the SK inner detector volume.

We generate MC samples for the three SN models for the three neutrino oscillation hypotheses: no oscillation, normal hierarchy (NH) and inverted hierarchy (IH). Table 1 shows the expected numbers of events of the three SN models at SK in the 22.5-kton fiducial volume with the total energy threshold of $7 \mathrm{MeV}$, obtained by averaging the 3,000 MC ensembles at the distance of $10 \mathrm{kpc}$. Figure 1 shows the reconstructed energy distributions at SK for the Wilson and NK1 models with and without neutrino oscillations to display the effect of the neutrino oscillations. Figure 1 also shows the energy spectrum of the spallation events found in the silent warnings described in Sec. 2.2.

\subsection{The monitor system}

In this section, we will describe details of the SN neutrino burst monitor system. Figure 2 shows a flow diagram of the system. The SK data collected by the data acquisition system are sent to the event builder. At the event builder, the event data are packed and stored in a data file which we call a sub-run file. Each sub-run file contains about one minute of event data. The sub-run files are sent to both the offline process and the SN burst monitor. In the offline process, the data files are converted to an offline data format that is used for various physics analyses and detector calibrations. The SN monitor system is running on a single computer on which a control process operating continuously handles all the processes and the data files.

For each sub-run file sent to the SN monitor, two processes are automatically executed by the control process: a reformat process (the first process) and an event reconstruction process (the second process). The reformat process converts the online data format to the offline data format. Using the offline format data, the event reconstruction process reconstructs the vertex position, direction and energy for each event. It takes about two minutes to finish the reformat and event reconstruction for one sub-run file.Events with total energy greater than $7 \mathrm{MeV}$ and vertex position within the 22.5-kton fiducial volume in SK are selected. We remove cosmic 
ray muons and their subsequent decay electron events.After the reconstruction of each selected event, a 20-second time window is opened backwards in time from the event, and the number of selected events in the window $\left(N_{\text {cluster }}\right)$ is counted. If there is a sub-run file boundary, the time window extends to the previous sub-run file.

We also compute a variable $D$ that characterizes the vertex distribution. The variable $D$ identifies the dimension of the vertex distribution and is an integer number from 0 to 3 , corresponding to point-, line-, plane- and volume-like distributions, respectively. The variable $D$ is determined by comparing $\chi^{2}$ values obtained from the lengths of the major and minor axes that correspond to the eigenvalues of a correlation matrix of the vertex distribution. The correlation matrix is a $3 \times 3$ matrix whose elements are defined as $\left\langle\left(x_{i}-\left\langle x_{i}\right\rangle\right)\left(x_{j}-\left\langle x_{j}\right\rangle\right)\right\rangle$, where $i, j=1,2,3$ identify the vertex position axes and $\langle x\rangle$ is the mean value of a variable $x$. We construct a $\chi^{2}=\sum_{n=1}^{N_{\text {cluster }}}\left|\vec{d}_{n}-\vec{d}(\vec{s})\right|^{2}$, where $\vec{d}_{n}$ is the $n$-th event vertex position and $\vec{d}(\vec{s})$ is a position closest to $\vec{d}_{n}$ on either a point, a line or a plane with parameters $\vec{s}$ that determine the geometry of the three cases. The three eigenvalues $\lambda_{i}\left(i=1,2,3\right.$, and $\left.\lambda_{1} \leq \lambda_{2} \leq \lambda_{3}\right)$ are used to construct the minimum $\chi^{2}$ values that are $\left(\lambda_{1}+\lambda_{2}+\lambda_{3}\right) / 3,\left(\lambda_{1}+\lambda_{2}\right) / 2$ and $\lambda_{1}$, computed by assuming the vertex distribution is point-, lineand plane-like, respectively, with the condition of $\partial \chi^{2} / \partial \vec{s}=0$. The comparison of the $\chi^{2}$ values to determine a $D$ value is tuned using MC simulations so that the calculated $D$ value reproduces the input one. In case of a real SN burst, the vertex distribution should be uniform in SK, and we would have $D=3$, depending on the number of burst events, which is confirmed by a simulation. In contrast, for the case of a background burst mainly originating from spallation events, the vertex positions distribute along the parent muon tracks, and we would have $D=2,1$ or 0 , where the spallation events are the radioactivities created by both high-energy cosmic ray muons and by constituents of the resulting hadronic showers. When the process finds $N_{\text {cluster }} \geq 60$ events and $D=3$, it generates a prompt $\mathrm{SN}$ burst warning which initiates phone-callings and emails sent to experts in the SK collaboration within a few minutes after the SN burst occurs. We call such a warning a "golden" warning. Subsequent to a golden warning, the experts start a meeting in order to make a world-wide announcement within one hour. The threshold of $N_{\text {cluster }}$ is determined so that we would have $100 \%$ SN detection efficiency at the Large Magellanic Cloud (LMC) assuming the three SN models described in Section 2.1.

The third process in Fig. 2 combines all the sub-run data and determines the SN direction by a fit. All the SN burst event information is summarized and sent to the experts by e-mail, which is also used as the input to the discussions. Following these discussions the announcement containing the information about the number of observed neutrinos, the burst time duration, the universal time the burst happens and the estimated direction of the SN in the equatorial coordinate system is broadcast to the ATEL [21], GCN [22], IAU-CBAT [23] and SNEWS [8]. The universal time is determined using 1 pps (pulse per second) signals from the global positioning system and a local time clock system consisting of a commercial rubidium clock [24]. No golden prompt warning has been sent so far.

When the SN burst has less than 60 events, the golden warning will not be generated. Instead we set another threshold. The threshold for generating the warning is determined so that the backgrounds are suppressed: we set the threshold of $N_{\text {cluster }} \geq 25$ and require $D=3$. The warning generated with this condition is called a normal warning. The normal warning is sent to the experts only by e-mail, without phone-calls, and to SNEWS. Conveners among the experts check the event cluster found by this warning and make a decision about whether to have a meeting for the announcement. The normal warning threshold is set so that we would have $100 \%$ SN detection efficiency at the Small Magellanic Cloud (SMC) assuming the three SN models. 
The details of the detection efficiency will be described in Section 2.3. The reason to provide the normal warning is to avoid any fake warnings caused by unexpected software and hardware troubles. We have had no normal warning so far.

In summary, the $\mathrm{SN}$ monitor reformats the data and determines the vertex position, direction, and energy of events within a few minutes of the data being collected. It then searches for bursts of events - a cluster occurring within 20 seconds - with energies above $7 \mathrm{MeV}$ and whose vertices fall within SK's 22.5-kton fiducial volume. Within an hour of a warning being issued, the experts gather and hold a meeting to determine the appropriate public announcement to make, if any, based on the quality and nature of the detected burst.

We also provide a lower threshold such that we require more than 13 events in 10 seconds. We call a warning generated with this condition a "silent" warning. The conditions were tuned so that we would have a few warnings per day from spallation events. The silent warnings are sent to only a few experts of the monitor system operation and detector condition, and are not used as the fast alert for a SN burst.

\subsection{Performance study of the $S N$ burst monitor with simulations}

Figure 3 shows the minimum $\chi^{2}$ distributions for the three geometrical assumptions and $D$ distributions for SN MC and spallation data triggered by the silent warnings. The spallation event cluster is identified from the vertex, energy and time distributions, i.e., the vertex distribution is concentrated around the parent muon track, the energies of the events have the typical spallation energy spectrum up to $20 \mathrm{MeV}$, and the time distribution is an exponential decay consistent with the lifetimes of the spallation products. In the figure, we generate MC simulation samples in the range of $60 \leq N_{\text {cluster }} \leq 100$ uniformly, and plot the distributions for the samples. The probability to have $D \leq 2$ for SN MC with the $N_{\text {cluster }}$ range is $8 \times 10^{-4}$. No SN MC sample having $D \leq 2$ is found for 930,000 samples with $100<N_{\text {cluster }}<1,000$. For a normal warning condition, i.e., $25 \leq N_{\text {cluster }}<60$, the probability to have $D \leq 2$ is $1.3 \%$. This demonstrates that the variable $D$ can discriminate between the $\mathrm{SN}$-like clusters and spallation background clusters.

Figure 4 shows the $\mathrm{SN}$ detection efficiency as a function of a distance to a $\mathrm{SN}$ for the normal and golden warnings for the three SN models without neutrino oscillation and with neutrino oscillations for normal and inverted mass hierarchy hypotheses. It is found that the system has $100 \%$ detection efficiency up to the LMC located at $50 \mathrm{kpc}$ away for all three models for the golden warning with the three hypotheses. For the SNe at the SMC, about $64 \mathrm{kpc}$ away, the efficiency depends on the hypotheses for the Nakazato model, and is 100\% for the Wilson model. The normal warning has almost $100 \%$ detection efficiency for the three models. The efficiency is basically determined by the number of inverse beta decay events. The difference between detection efficiencies among the three hypotheses of the neutrino oscillations is caused by the difference between the $\bar{v}_{e}$ energy spectra. The average energy of $\bar{v}_{e}$ is smaller than that of $\bar{v}_{x}$ when those neutrinos are emitted from the neutrinosphere. With neutrino oscillations, $\bar{v}_{x}$ are converted to $\bar{v}_{e}$ and therefore the average energy of $\bar{v}_{e}$ at SK increases, resulting in a higher event rate of the inverse beta decays. Due to this effect, the detection efficiency of SNe at the SMC increases for the case of neutrino oscillations.

\subsection{Operation of the SN burst monitor}

We have operated the SN burst monitor system for about 20 years, since the beginning of SK data-taking in 1996. The SN burst selection criteria and operation scheme have been changed, updated, and improved throughout this period. The SN monitor system scheme described in this 
report came into service in April of 2013. Before that time, earlier versions of the monitor system had been running as one of the offline processes.

In Fig. 5 (a), we show the silent warning rates per 24 hours as a function of the elapsed days from Jan. 1st, 2010. The rate has trends as a consequence of - and which track - variations of the water transparency in SK. The energy scale used in the energy reconstruction program in the SN monitor process has been continually adjusted to compensate for these transparency fluctuations. Despite these fluctuations, the warning rate has been relatively stable over the last six years, with an average rate of 2.4 warnings per 24 hours.

The expected number of accidental background events satisfying the event selection is 0.121 events per 10 seconds with a root mean square of 0.007 events. We estimate this by counting the number of events in the SK fiducial volume with a total energy greater than $7 \mathrm{MeV}$ for one day, and scale this number to a rate per 10 seconds. Figure 5 (b) shows the estimated averaged background event rate for a recent period of 434 days. The background events are considered to be spallation products, since there should be negligible contamination from known radioactivities other than spallation products given the applied energy threshold.

Figure 5 (c) shows the data processing time distribution for the silent warnings found. The average time to finish the processing is about 170 seconds; fluctuations are caused by the reconstruction process and the condition of the network through which the data sample files are copied from the SK data acquisition system. The offline SN monitor that had run before April 2013 took about five minutes to finish the reformat and reconstruction processes, as the offline reconstruction program was tuned for physics analysis and calibration. We have optimized the reconstruction program for the online SN monitor to increase the processing speed without degrading its performance.

Figure 5 (d) shows the averaged monthly duty cycle of the SN monitor system over a recent 34month period; the SN monitor operates with a duty cycle of about $97 \%$. The monitor searches for SN event bursts during normal SK running, but it does not operate during SK detector calibration runs, particularly during those calibration runs employing artificial sources that intentionally generate event bursts. Most of the $3 \%$ loss of the SN monitor duty cycle comes from planned calibration. Note that even when the SN monitor is off, SK still has a non-realtime capability to detect a SN burst during these calibration runs. This is achieved via dedicated offline analyses which remove likely source events based on their vertex positions and event timings.

We use the spallation events found as silent warnings to estimate the false alarm rate by assuming constant rate Poisson processes rather than generating simulation samples of the backgrounds. We combine multiple silent warnings randomly and form a combined cluster to estimate a probability of having a golden (normal) warning. Using 2,551 silent warnings, we combined two of them for all combinations of two spallation bursts, to form ${ }_{2551} C_{2}=3,252,525$ patterns, and estimate the probability for the combined burst to pass the criteria for a golden (normal) warning to be $0\left(4.3 \cdot 10^{-6}\right)$. For three combinations with ${ }_{2551} C_{3}$ patterns, the probability is estimated to be $4.1 \cdot 10^{-5}\left(1.9 \cdot 10^{-4}\right)$ for a golden (normal) warning. One silent warning happens every 10 hours. The probability to have two (three) spallation clusters coincident within $20 \mathrm{sec}$ is $5.6 \cdot 10^{-4}\left(3.1 \cdot 10^{-7}\right)$. Therefore the probability to have a golden (normal) warning due to accidentally coincident spallation bursts is $3.1 \cdot 10^{-7} \times 4.1 \cdot 10^{-5}=1.3 \cdot 10^{-11}\left(5.6 \cdot 10^{-4} \times 4.3 \cdot 10^{-6}=\right.$ $\left.2.4 \cdot 10^{-9}\right)$. The false alarm rates are calculated to be once per $9.0 \cdot 10^{7}$ years for a golden warning and once per $4.7 \cdot 10^{5}$ years for a normal warning.

The processes in the $\mathrm{SN}$ burst monitor are kept under observation by a web-based monitor running on a dedicated PC. Any problems are displayed on the web monitor immediately after they are found, and the SK shift takers are notified by visible and audio alerts. 


\section{Determination of the SN direction}

The determination of the $\mathrm{SN}$ direction is crucial since the direction information provided by neutrinos is useful for astronomers to observe the $\mathrm{SN}$ explosion process from the onset via electromagnetic waves. At present, SK is the only operating experiment with sufficient detector mass to determine the neutrino direction from elastic scattering events which preserve the SN direction. Though the inverse beta decay events also have a correlation with the SN direction, the elastic scattering events mainly dominate the direction determination power. A study of the determination of the SN direction using neutrinos was performed by [25][18]. Here, we present a method we have developed. We will explain the algorithm to determine the SN direction, and then will show its performance obtained using SK MC.

\subsection{Algorithm}

We determine the SN direction based on a maximum likelihood method. A likelihood function $L_{i}$ for $i$-th event is defined as:

$$
L_{i}=\sum_{r} N_{r k} p_{r}\left(E_{i}, \hat{d}_{i} ; \hat{d}_{\mathrm{SN}}\right)
$$

where the index $r$ indicates one of the four neutrino interaction channels: inverse beta decay $\left(\bar{v}_{e} p\right)$, electron elastic scattering of anti-electron neutrino $\left(\bar{v}_{e} e\right)$, other elastic scatterings $(v e)$ and the charged-current interactions on oxygen $\left(v^{16} \mathrm{O}\right)$. The index $k$ indicates the energy bin, running from 1 to 5 for the energy ranges of $7<E<10,10<E<15,15<E<22,22<E<35$ and $35<E<50$, respectively, where $E$ is the measured total electron energy in MeV. $N_{r k}$ is the number of events of the interaction $r$ in the $k$-th energy bin. $E_{i}$ is the $i$-th event total electron energy, which uniquely determines the index $k, \hat{d}_{i}$ is the $i$-th event direction and $\hat{d}_{\mathrm{SN}}$ is the SN direction we want to determine. The $p_{r}\left(E_{i}, \hat{d}_{i} ; \hat{d}_{\mathrm{SN}}\right)$ function is a probability density function (PDF) for interaction $r$ as a function of the energy $E_{i}$ and an inner-product of $\hat{d}_{i} \cdot \hat{d}_{\mathrm{SN}}=\cos \theta_{\mathrm{SN}}$. The PDF is determined using SK MC. The number of $\bar{v}_{e}$ elastic scattering events can be inferred from the number of inverse beta decay events with the relation $N_{\bar{v}_{e} e, k}=\sum_{m} A_{k m} N_{\bar{v}_{e} p, m}$, where the matrix $A_{k m}$ is calculated from a ratio of the total cross sections of the two interactions. We determine PDFs for elastic scatterings with the following procedure. We divide the SN MC sample elastic scattering events generated with the Wilson model into one-MeV bins from 7 to $35 \mathrm{MeV}$. For energies greater than $35 \mathrm{MeV}$, we combined all events into one bin. Then we fit the $\cos \theta_{\mathrm{SN}}$ distribution with the known SN direction in MC using a model function that is the superposition of four exponential functions and containing eight parameters for each energy bin. For a given energy value, we compute the eight parameter values by interpolating the parameter values of neighboring two energy bins and applying those to the model function to obtain the $\mathrm{PDF}$ value. A similar procedure is applied to the PDFs for inverse beta decays and interactions on oxygen to determine the PDF values. We construct a likelihood $\mathcal{L}=\exp \left(\sum_{k, r} N_{r k}\right) \prod_{i} L_{i}$, and maximize it so that:

$$
\frac{\partial \mathcal{L}}{\partial N_{r k}}=\frac{\partial \mathcal{L}}{\partial \hat{d}_{\mathrm{SN}}}=0,
$$

where for $N_{r k}$ we vary $r=\bar{v}_{e} p$, ve and $v^{16} \mathrm{O}$. For $r=v^{16} \mathrm{O}$, we assume the $\cos \theta_{\mathrm{SN}}$ is same for neutrino and anti-neutrino interactions. We set $N_{r k}=0$ for $r=v^{16} \mathrm{O}$ with $k=1,2,3$, as the expected number of charged current interactions on oxygen is negligible in those energy ranges, as shown in Fig. 1.The SN direction $\hat{d}_{\mathrm{SN}}$ contains two parameters: zenith and azimuth angles, 
that are translated to the direction in the equatorial coordinate system with the time the burst is found. Hence, we vary 14 parameters of $N_{r k}$ and $\hat{d}_{\mathrm{SN}}$ in total.

When we perform a fit with the likelihood method, we first determine the initial value of the direction based on a grid search: we scan all $\hat{d}_{\mathrm{SN}}$ to the $4 \pi$ directions with a coarse grid step and count the number of events that satisfy $\cos \theta_{\mathrm{SN}}>0.8$ at each step, and we set the initial value that gives the largest number of events.

\subsection{Performance}

Figure 6 demonstrates $\cos \theta_{\mathrm{SN}}$ distributions of a fit to a MC sample of the Wilson model at $10 \mathrm{kpc}$ for the five energy bins and combined one with the superpositions of the fitted likelihood functions. Figure 7 shows the corresponding direction distribution on a sky map in the equatorial system. The red (blue) points are the reconstructed directions of each elastic scattering event (inverse beta decay or charged current reaction on oxygen), and the star mark shows the reconstructed SN direction. The elastic scattering events concentrate around the reconstructed SN direction, while the distribution of inverse beta decays and charged current events is almost uniform across the entire sky.

Figure 8 shows $\Delta \theta$ distributions of the three models (Wilson, NK1 and NK2) for 3,000 MC samples at $10 \mathrm{kpc}$ without neutrino oscillation, where $\Delta \theta$ is the angle between the input $\mathrm{SN}$ direction and the fitted direction. The solid lines are fit results using the von Mises-Fisher (MF) distribution [26]:

$$
f(\Delta \theta ; \kappa)=\frac{\kappa}{2 \sinh \kappa} e^{\kappa \cos \Delta \theta} \sin \Delta \theta,
$$

where $\kappa$ determines the sharpness of the distribution concentration on a sphere.

We estimate the angular resolutions of the $\mathrm{SN}$ direction determination using an ensemble estimation. In order to cope with any possible combinations of the elastic scatterings and inverse beta decays, we employ the following method. We generate a number of MC samples for various combinations of fitted yields of the elastic scatterings and inverse beta decays in the ranges up to 1,500 for the former and 60,000 for the latter. We divide each range into 15 to obtain a $15 \times 15$ matrix. Each matrix element contains about 3,000 MC samples. For each element, we determine the angle $\theta_{\text {en }}$ that covers $68.2 \%, 90 \%$ and $95 \%$ of the MC samples. We also provide probabilities to have the true $\mathrm{SN}$ position in 2, 5 and 10 degrees with respect to the fitted direction. We generate the matrices for the Wilson and NK1 models with $(\mathrm{NH})$ and without neutrino oscillations. The dependence of the $\theta_{\mathrm{en}}$ on the models has about a $10 \%$ variation. We employ the largest values of $\theta_{\text {en }}$ and the smallest value of the probabilities for each matrix element conservatively. When we find a SN neutrino burst, we apply the fit to the burst events to obtain the SN direction and the yields of the elastic scatterings and inverse beta decays. With the fitted yields, we identify the matrix element and obtain the angular resolutions and the probabilities that are to be announced to the public.For example, we find $\theta_{\mathrm{en}}=3.1 \sim 3.8^{\circ}\left(4.3 \sim 5.9^{\circ}\right)$ at $68.2 \%$ coverage for the Wilson (NK1) model at $10 \mathrm{kpc}$, where the range covers various neutrino oscillation scenarios in Table 1.

The Nakazato model also provides a SN model with a black hole formation for $M=30$ solar mass [13]. The model predicts that neutrino emission suddenly stops $842 \mathrm{~ms}$ after the core bounce. If the SN monitor observes an abrupt cutoff of the supernova neutrino flux, this could be the signature of the birth of a black hole.Based on this model, we generate MC samples and apply the fit. We obtain an angular resolution of 2.3 degrees at $10 \mathrm{kpc}$ with $68.2 \%$ confidence level. Therefore this may help the identification of a position of a disappeared massive star as proposed by [27]. 
In order to understand the behavior of the estimated angular resolution, we make use of the curvature of the likelihood at its maximal position: we define a value $\sigma$ as:

$$
\sigma=\sqrt{-\frac{1}{\frac{\partial^{2} \ln \mathcal{L}}{\partial \theta_{\mathrm{SN}}^{2}}}}
$$

where the second derivative is the curvature calculated at the point on the sphere at which the likelihood function $\mathcal{L}$ becomes maximal. We calculate $\sigma$ values along four planes that include the maximal point and have different azimuth angles with respect to the point, and employ the maximum among the four $\sigma$ values. Then we obtain the resolution $\theta_{\sigma}(q)$ that covers an area with a fraction of $q=1-p$ of the MF distribution with a $p$ value:

$$
\theta_{\sigma}(q)=\arccos \left[\frac{1}{\kappa} \ln \left(1-q+q e^{-2 \kappa}\right)+1\right],
$$

and $\kappa=1 / \sigma^{2}$.

In Fig. 9 (a) and (c), we show the obtained angular resolution at the $68.2 \%$ coverage for the ensemble estimation of the fit, the likelihood curvature method and the ensemble estimation using a grid search as a function of the distance for the Wilson and NK1 models. We find that the $\Delta \theta$ distributions are well modeled by the MF function and the likelihood curvature estimation $\theta_{\sigma}(q)(q=0.682)$ is consistent with that of the ensemble estimation for the statistics larger than those of $\mathrm{SNe}$ at $10 \mathrm{kpc}(7 \mathrm{kpc})$ for the Wilson (NK1) model. However, $\theta_{\sigma}(q)$ is found to be an underestimate for the smaller statistics. The degradation of the angular resolution for the distant $\mathrm{SNe}$ is likely due to failure in giving a proper initial value of the SN direction by the grid search, indicated by the fact that the $\theta_{\text {en }}$ value approaches that of the grid search for larger distances (smaller statistics). The small statistics produce a large fluctuation that sometimes makes a fake peak on the grid direction search. That makes the initial value a wrong direction and the likelihood fit finds a local minimum around the direction. Figure 9 (b) and (d) show the ensemble estimation of the angular resolution as a function of the SN distance for three neutrino oscillation hypotheses. The angular resolutions with the two neutrino oscillation hypotheses are smaller than those without neutrino oscillation. This is due to an increase in elastic scattering events as shown in Table 1.

We estimate the precision of the angular uncertainty using Eq. (5) under the assumptions of the SN models used; the precision of $\Delta \theta_{\sigma}$ is determined using $\Delta \theta_{\sigma}=d \theta_{\sigma} / d q \cdot \Delta q=d \theta_{\sigma} / d q \cdot \sqrt{q / N}$, where $N=3,000$ is the number of samples in the ensemble. We find $\Delta \theta_{\sigma}=0.09$ degrees with $q=0.682$ for the NK1 model with the NH neutrino oscillation at a distance of $10 \mathrm{kpc}$, which is much smaller than the angular resolution variation of various neutrino oscillation scenarios.

\section{Summary}

We describe a real-time monitor of a SN neutrino burst at SK. The monitor is able to provide a fast warning to the world within one hour. The system is operating on a dedicated computer independent of the offline processes. The SN neutrino burst selection criteria are determined so that fake event bursts mainly caused by spallation events are rejected. Using MC simulations, we find that the system has $100 \%$ detection efficiency up to the LMA for the three SN models with the golden warning criteria. The expected total number of neutrino events with the selection 
criteria is about 5,200 $(2,200)$ for the Wilson (NK1) model at $10 \mathrm{kpc}$ without neutrino oscillation. Neutrino oscillations increase the number of inverse beta decay events and enhance the detection efficiency for SNe at the SMC for both normal and inverted hierarchy hypotheses.

The SN direction pointing is of importance as it provides a chance for astronomers to observe the SN explosion from its onset with electromagnetic waves. SK is the only detector that enables us to reconstruct the SN direction using only neutrinos among the existing neutrino observatories. We have developed an algorithm to identify the $\mathrm{SN}$ direction and its error using a maximum likelihood method. The pointing accuracy estimated by the ensemble study is found to be $3.1 \sim$ $3.8^{\circ}\left(4.3 \sim 5.9^{\circ}\right)$ at $68.2 \%$ coverage for the Wilson (NK1) model at $10 \mathrm{kpc}$, where the range covers various neutrino oscillation scenarios.

\section{Acknowledgements}

We gratefully acknowledge the cooperation of the Kamioka Mining and Smelting Company. The Super-Kamiokande experiment has been built and operated from funding by the Japanese Ministry of Education, Culture, Sports, Science and Technology, the U.S. Department of Energy, and the U.S. National Science Foundation. Some of us have been supported by funds from the Research Foundation of Korea (BK21 and KNRC), the Korean Ministry of Science and Technology, the National Research Foundation of Korea (NRF- 20110024009), the European Union (H2020 RISE-GA641540-SKPLUS), the Japan Society for the Promotion of Science, the National Natural Science Foundation of China under Grants No. 11235006, the National Science and Engineering Research Council (NSERC) of Canada, and the Scinet and Westgrid consortia of Compute Canada. This work was partly supported by the Grant-in-Aid for Scientific Research on Innovative Areas [JSPS No.26104006].

\section{References}

[1] K. Hirata et al., Phys. Rev. Lett. 58, 1490 (1987); R. M. Bionta et al., Phys. Rev. Lett. 58, 1494 (1987); E. N. Alekseev, L. N. Alekseeva, V. I. Volchenko and I. V. Krivosheina, JETP Lett. 45, 589 (1987); M. Aglietta et al., Europhys. Lett. 3, 1315 (1987).

[2] In this paper, according to particle-physics nomenclature, "event" refers to a neutrino interaction recorded by Super-Kamiokande.

[3] D. N.Schramm and J. W. Truran, Phys. Rept. 189, 89 (1990); M. Koshiba, Phys. Rept. 220, 229 (1992).

[4] S. M. Adam et al., Astrophys. J. 778, 164 (2013).

[5] T. Shigeyama, K. Nomoto, M. Hashimoto and D. Sugimoto, Nature 328, 320 (1987); S. Blinnikov et al., Astrophys. J. 532, 1132 (2000).

[6] R. C. Svoboda, NASA-CR-194367 (1992); Y. Oyama et al., Nucl. Instr. Meth. A 340, 612 (1994); M. Ambrosio et al., Astropart. Phys. 8, 123 (1998).

[7] E. N. Alexeyev and L. N. Alexeyeva, J. Exp. and Theo. Phys. 95, 5 (2002); M. E. Monzani et al., Nuovo Cim. C 29, 269 (2006); N. Yu. Agafonova et al., Astropart. Phys. 28, 516 (2008); A. A. Aguilar-Arevalo et al., Phys. Rev. D 81, 032001 (2010); R. Abbasi et al., A\&A 535, A109 (2011); H. Wei et al., arXiv:1310.5783; K. Asakura et al., arXiv:1506.01175; K. Zuber, Nucl. Part. Phys. Proc. 265-266, 233 (2015).

[8] P. Antonioli et al., New J. Phys. 6, 114 (2004); K. Scholberg, Astron. Nachr. 329, 337 (2008).

[9] S. Fukuda et al., Nucl. Instrum. Meth A 501, 418 (2003).

[10] H. Nishino et al., Nucl. Instrum. Meth A 610, 710 (2009).

[11] S. W. Li and J. F. Beacom, Phys. Rev. C 89, 045801 (2014).

[12] T. Totani et al., Astrophys. J. 496, 216 (1998).

[13] K. Nakazato, K. Sumiyoshi, H. Suzuki, T. Totani, H. Umeda and S. Yamada, Astrophys. J. Supp. 205, 3 (2013)

[14] A. S. Dighe and A. Y. Smirnov, Phys. Rev. D 62, 033007 (2000).

[15] Particle Data Group (the 2014 edition), http://pdg. lbl.gov/.

[16] P. Vogel and J. F. Beacom, Phys. Rev. D 60, 053003 (1999). 
[17] E. Kolbe, K. Langanke, and P. Vogel, Phys. Rev. D 66, 013007 (2002).

[18] R. Tomas, D. Semikoz, G. G. Raffelt, M. Kachelrieß and A. S. Dighe, Phys. Rev. D 68, 093013 (2003).

[19] J. N. Bahcall, M. Kamionkowski and A. Sirlin, Phys. Rev. D 51, 6146 (1995).

[20] K. Abe et al., Nucl. Instr. Meth, A737C, 253 (2014).

[21] The Astronomer's Telegram, http://www . astronomerstelegram.org/.

[22] The Gamma-ray Coordinates Network, http://gcn.gsfc.nasa.gov/.

[23] IAU: Central Bureau for Astronomical Telegrams, http://www. cbat. eps .harvard.edu/.

[24] K. Abe et al., Nucl. Instr. Meth. A 659, 106 (2011).

[25] J. F. Beacom and P. Vogel, Phys. Rev. D 60, 03307 (1999); S. Ando and K. Sato, Prog. Theo. Phys. 107, 957 (2002).

[26] R. A. Fisher, Proc. Roy. Soc. London Ser. A, 217, 295-305 (1953); K. V. Mardia and P. E. Jupp, "Directional Statistics", Wiley, (1972).

[27] C. S. Kochanek, et al., Astrophys. J. 684, 1336-1342 (2008). 

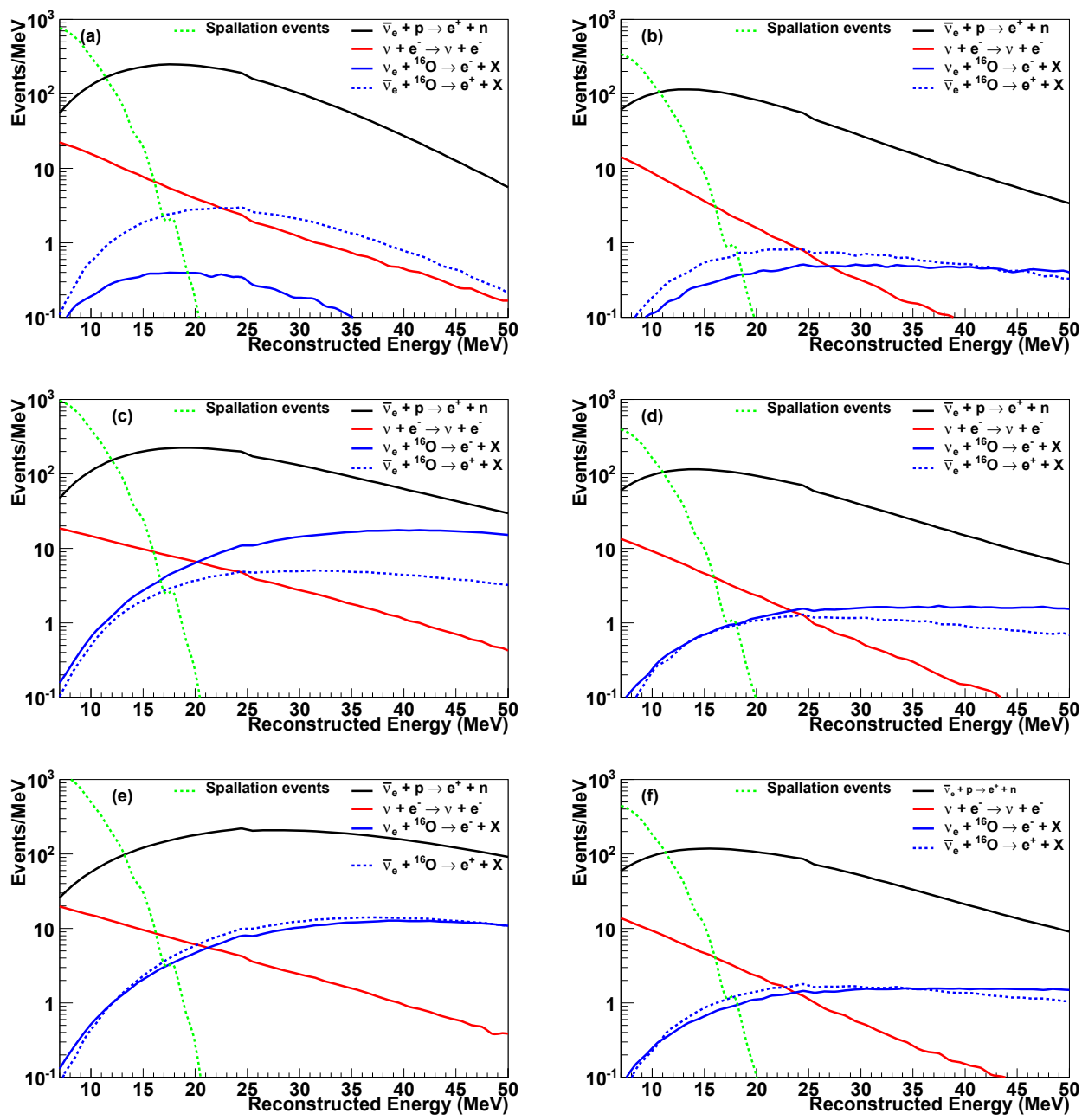

Figure 1: Reconstructed energy distributions at SK for (a) the Wilson model and (b) the NK1 model at $10 \mathrm{kpc}$ for the four neutrino interaction channels without neutrino oscillation, (c) the Wilson model and (d) the NK1 model with the NH hypothesis for neutrino oscillations, and (e) the Wilson model and ( $\mathrm{f}$ ) the NK1 model with the IH hypothesis for neutrino oscillations. The green dotted line is the energy spectrum of the spallation candidates found in the silent warnings. The spallation event histogram is normalized to the number of SN MC entries. 


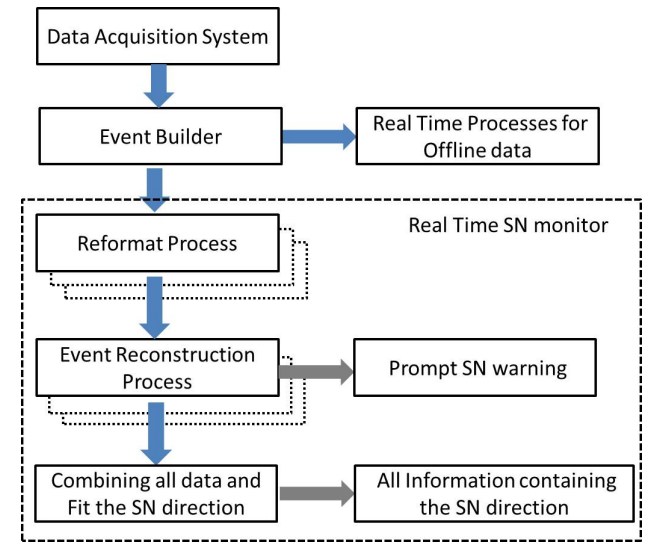

Figure 2: Block diagram of the real time SN neutrino burst monitor. The arrows show paths of the data flow, and boxes indicate processes. Details are explained in the text.
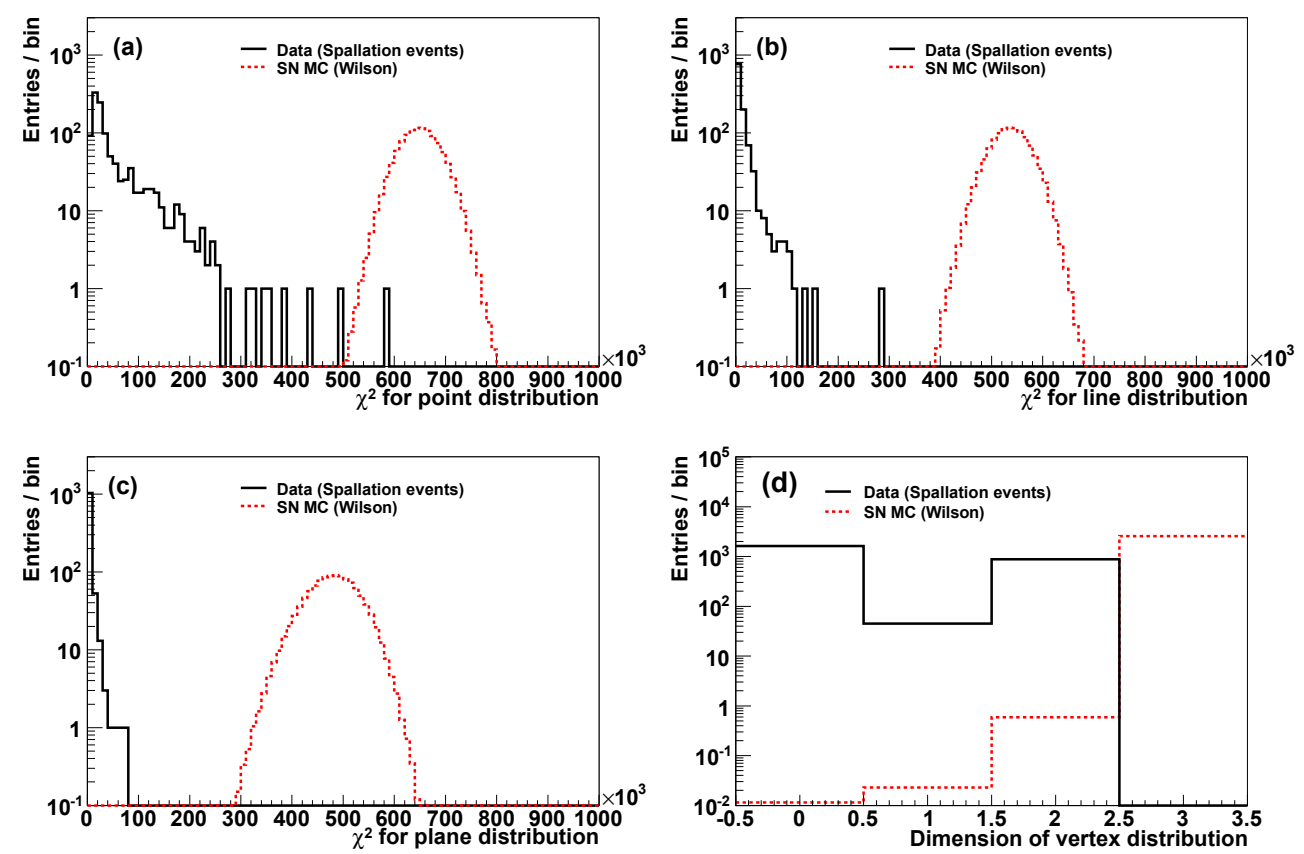

Figure 3: Minimum $\chi^{2}$ value distributions assuming (a) a point distribution, (b) a line distribution and (c) a plane distribution, and(d) distribution of $D$ (dimension of vertex distribution) for SN MC (red broken line) and spallation data (black solid line) found as silent warnings. For SN MC clusters, we plot the distributions for the MC samples with $60 \leq N_{\text {cluster }} \leq 100$. The histogram of SN MC is normalized to the number of data entries. 

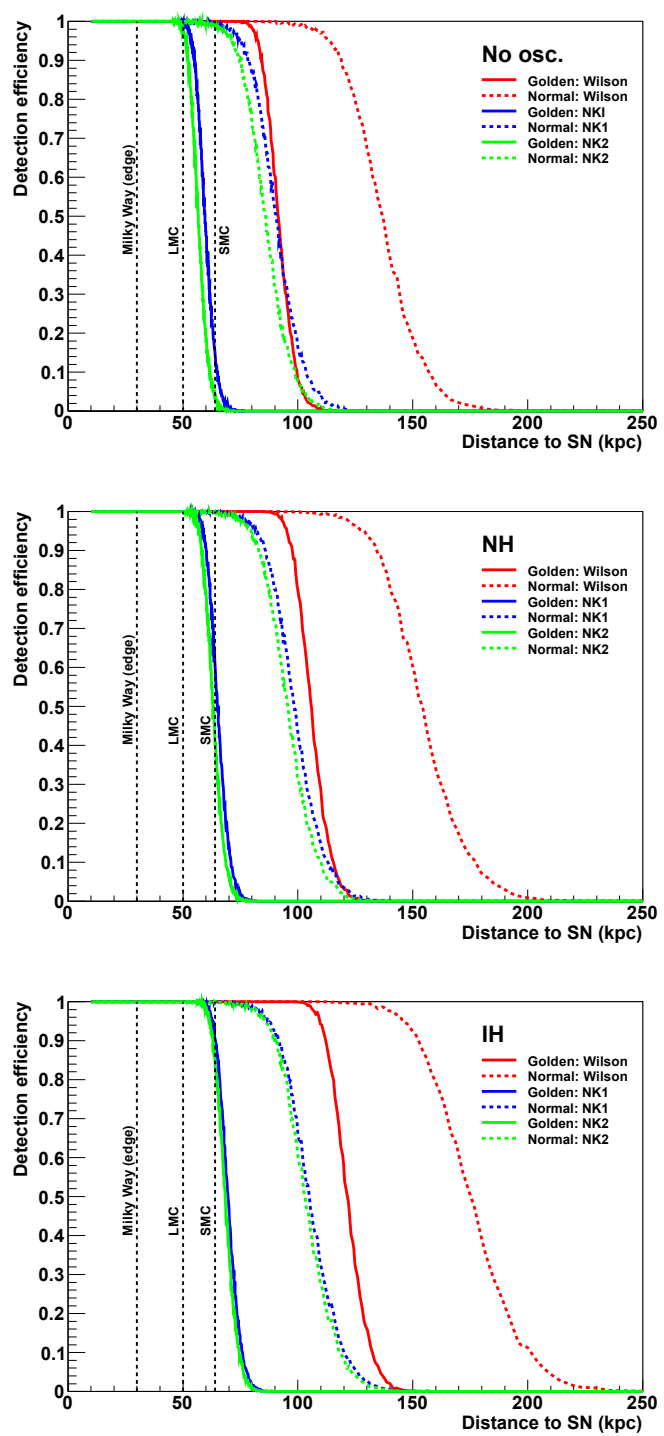

Figure 4: Detection efficiency of a SN burst as a function of a distance for the normal and golden warnings with the Wilson and Nakazato models. From top to bottom, without neutrino oscillation, with neutrino oscillations for the normal hierarchy and with neutrino oscillations for the inverted hierarchy. 

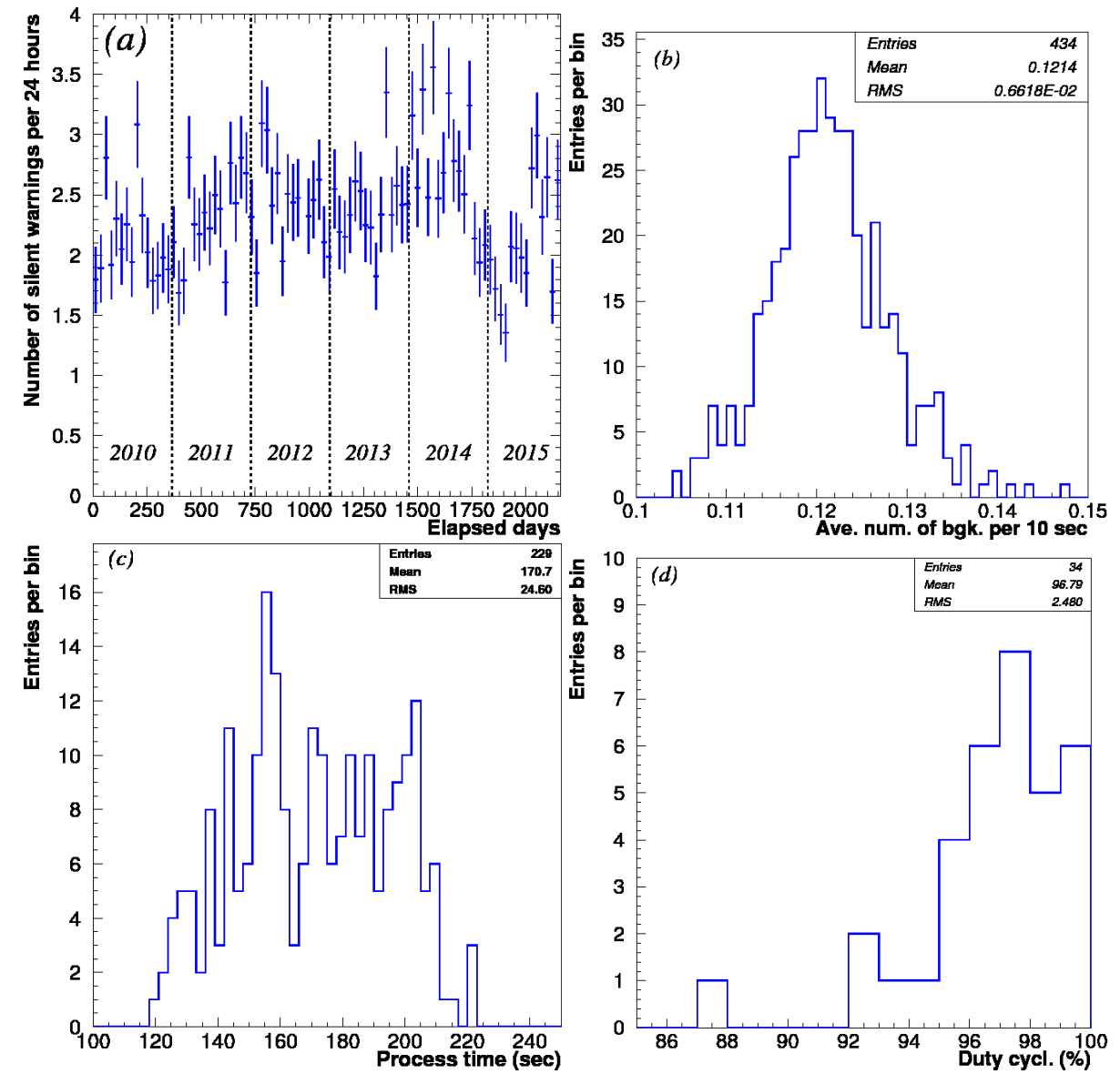

Figure 5: Stability of the SK operation: (a) the silent warning rate per 24 hours as a function of elapsed days from Jan. 1st of 2010, (b) averaged number of accidental background events per 10 seconds with one histogram entry for one day, (c) process time to finish the reformat and reconstruction to issue the silent warnings found, and (d) SN monitor duty cycle averaged over one month for each entry. 

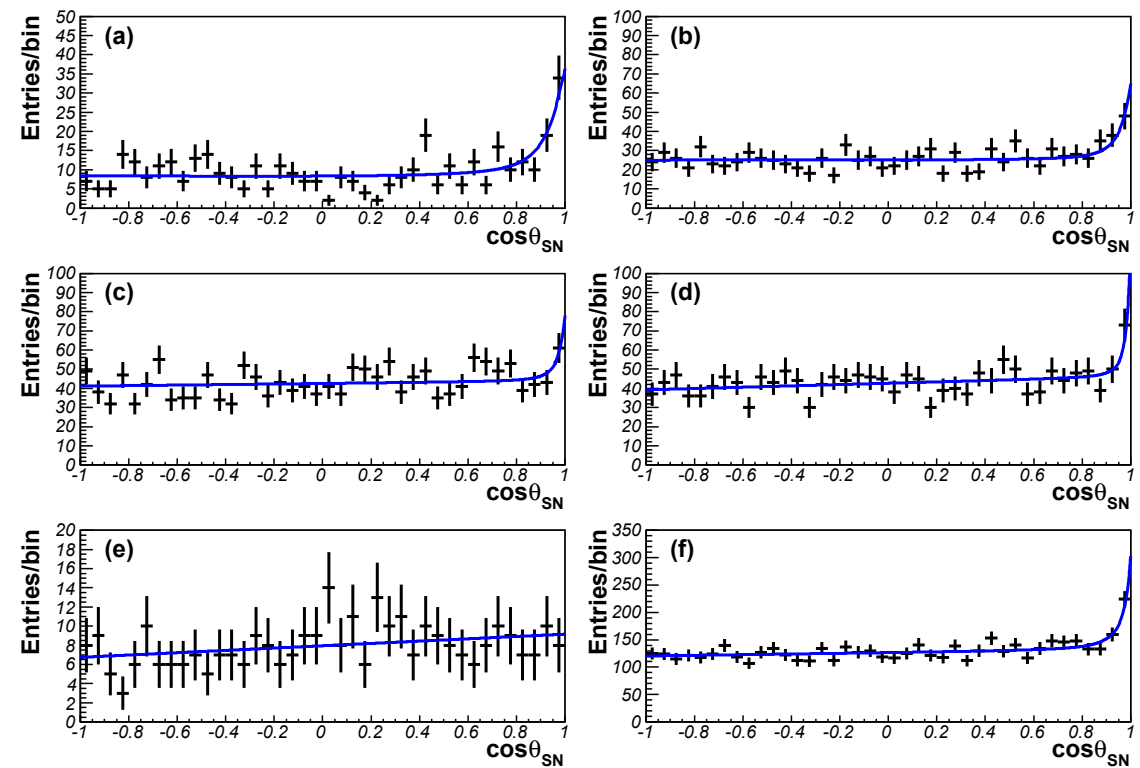

Figure 6: Distributions of $\cos \theta_{\mathrm{SN}}$ with the Wilson model at $10 \mathrm{kpc}$ for the five energy bins: (a) $7<E<10 \mathrm{MeV}$, (b) $10<E<15 \mathrm{MeV}$, (c) $15<E<22 \mathrm{MeV}$, (d) $22<E<35 \mathrm{MeV}$ and (e) $35<E<50 \mathrm{MeV}$, where $E$ is the measured total electron energy, and (f) all energies combined. The superimposed solid lines are the fitted likelihood functions.

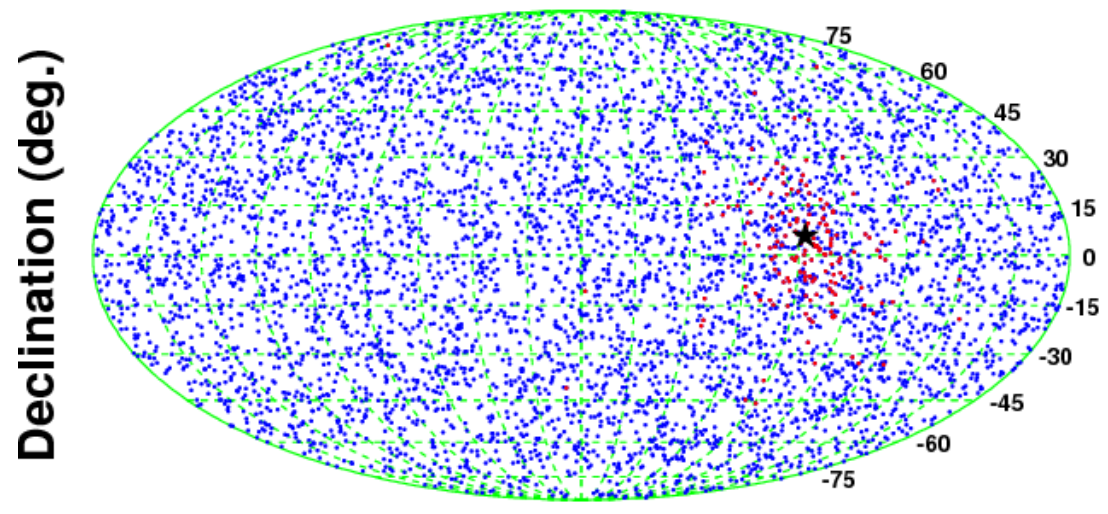

Right ascension (deg.)

Figure 7: Reconstructed direction on a sky map in the equatorial system obtained by a MC simulation with the Wilson model at $10 \mathrm{kpc}$. Red points are the directions of elastic scattering events, blue points are event directions of inverse beta decay and charged currents on oxygen, and the star point is the reconstructed SN direction. 


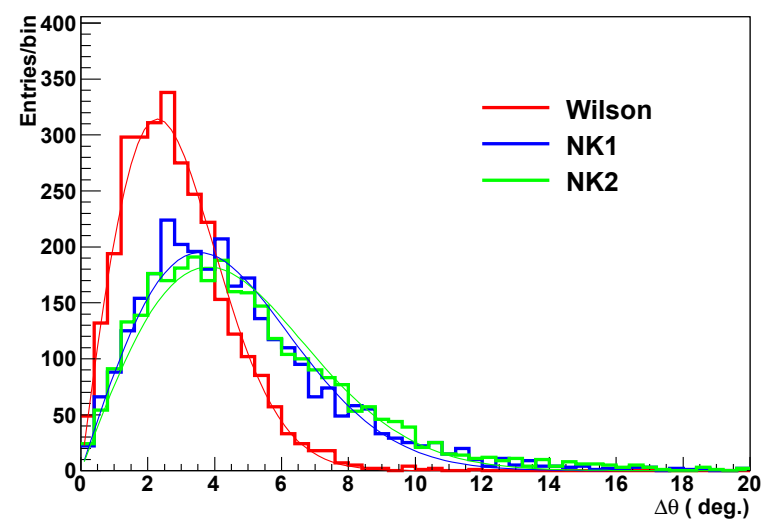

Figure 8: Distributions of $\Delta \theta$ for three models at a distance of $10 \mathrm{kpc}$ without neutrino oscillation. The superimposed solid lines are the fitted MF functions.
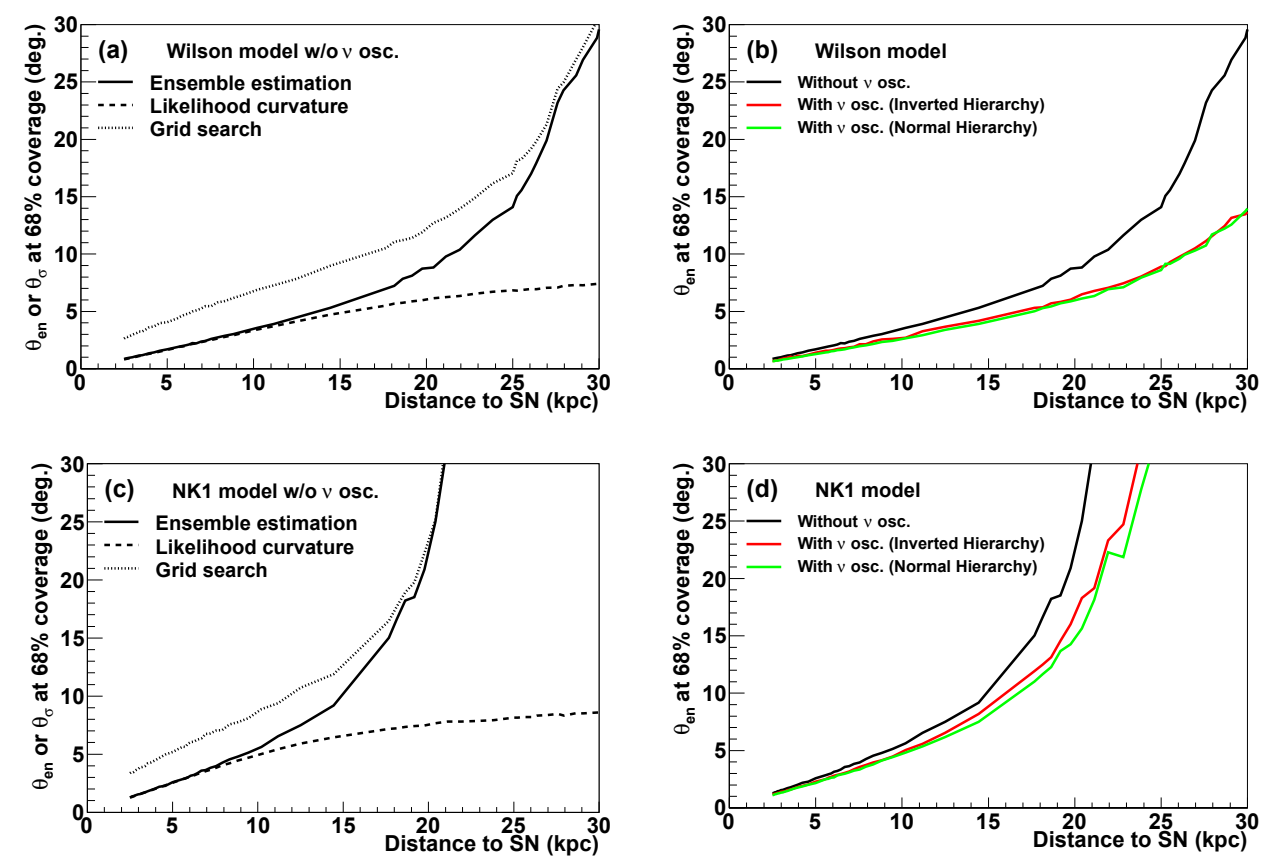

Figure 9: $\Delta \theta$ that covers $68 \%$ of $\mathrm{SNe}$ (the area) as a function of distance. In (a) and (c), the solid, dashed and dotted lines correspond to $\theta_{\text {en }}$ obtained by the ensemble estimation, $\theta_{\sigma}(0.682)$ obtained by the likelihood curvature with Eq.(5), and that obtained by the grid search, respectively, for the Wilson (a) and NK1 (c) models. Figure (b) and (d) show $\theta_{\text {en }}$ as a function of distance for the three neutrino oscillation hypotheses for Wilson (b) and NK1 (d) models. 